\title{
Recomendaciones para el análisis de datos acumulados de susceptibilidad antimicrobiana en instituciones de salud
}

\author{
Comité de Microbiología, Sociedad Chilena de Infectología*
}

*Miembros: Dona Benadof $F$ (Hospital Roberto del Río y Hospital DIPRECA, Santiago), Marcela San Martín S. (Clínica Vespucio, Santiago), Paola Pidal M. (Hospital Padre Hurtado, Santiago), Andrea Sakurada Z. (Hospital Dr. Sótero de

Río y Hospital Clínico Universidad de Chile, Santiago), Francisco Silva

O. (Hospital Clínico Universidad de Chile, Santiago), Cecilia Tapia P. (Clínica Dávila, Santiago e Instituto de Ciencias Biomédicas, Facultad de Medicina, Universidad de Chile Santiago), M. Teresa Ulloa F. (Instituto de Ciencias Biomédicas, Facul-

tad de Medicina, Universidad de Chile, Santiago), Maggie Vecchiola

H. (Hospital San José, Santiago) y Rossana Camponovo C. (Laboratorio ELSA, Santiago, revisora invitada)

Declaración: los autores declaran no tener conflictos de interés.

Recibido: 7 de diciembre de 2009

Correspondencia a: Dona Benadof Fuentes donaben@gmail.com

\section{Introducción}

C onsiderando la gran variabilidad en la distribución de la resistencia microbiana, se hace necesario que cada centro de salud genere localmente reportes de datos acumulados de susceptibilidad, con el propósito de guiar las decisiones clínicas y detectar tendencias que permitan establecer medidas para evitar la diseminación de cepas resistentes ${ }^{1,2}$.

La información generada debe ser lo suficientemente sólida como para permitir comparaciones en el tiempo y entre instituciones de salud, por lo que es importante adoptar metodologías que aseguren la calidad de los resultados.

Esta guía tiene como objetivo entregar recomendaciones especificas para el análisis de susceptibilidad antimicrobiana y aportar datos útiles para la elaboración del informe local. Se basó en la revisión del documento "Analysis and Presentation of Cumulative Antimicrobial Susceptibility Test Data" del Clinical and Laboratory Standards Institute (CLSI) ${ }^{3}$.

A continuación se mencionan los aspectos más importantes a considerar relacionados con la recolección, procesamiento de los datos, análisis de la información y presentación de los resultados.

\section{Recomendaciones generales}

El análisis de datos acumulados de susceptibilidad antimicrobiana se debe realizar al menos anualmente, especialmente si son utilizados para la elaboración de guías de terapias empíricas. Se recomienda analizar datos con mayor frecuencia si se evidencia un gran número de aislados, cuando se incorpora un nuevo antimicrobiano al estudio de susceptibilidad o cuando se perciben cambios clínicos epidemiológicos importantes.

Incluya sólo aislados clínicos y no de vigilancia y analice especies con al menos 30 aislados.

Calcule el porcentaje de sensibilidad (S) no incluyendo categoría intermedia (I), con algunas excepciones que se mencionan a lo largo de este artículo.

Incluya sólo el primer aislado por paciente en el período analizado, independientemente del sitio anatómico del cual se obtuvo y del patrón de susceptibilidad. Es importante tener en consideración que, según lo mencionado anteriormente, el aporte de esta metodología está orientada como guía para el tratamiento antimicrobiano empírico inicial.

\section{Requerimientos de la información}

El paso inicial es definir de dónde se va obtener la información y qué elementos se deben considerar. En 
cada uno de los ítems relacionados con los requerimientos de la información, existen aspectos considerados como indispensables que deben ser incluidos siempre en el momento de la recolección de datos y optativos, aquellos que se sugiere incorporar para enriquecer el análisis.

La recolección de los datos puede ser informatizada o realizarse en forma manual, considerándose esencial que sean obtenidos desde registros de laboratorio confiables donde la información esté sistematizada.

Información demográfica del paciente: Se considera indispensable la utilización de un número único en la identificación de paciente (por ejemplo: RUT), nombre, apellidos, y la procedencia: ambulatorio u hospitalizado. Es optativo incluir: servicio clínico, edad, diagnósticos, terapia antimicrobiana etc.

Información de muestras: Es indispensable incluir el tipo de muestra, fecha de recolección y número identificador único de muestra. Es optativo incluir el sitio anatómico de obtención.

Información del microorganismo: Es indispensable la identificación del microorganismo, preferentemente género y especie. Utilice el género cuando la especie no esté disponible. Es optativo incluir información relacionada con la interpretación clínica del resultado, como por ej.: colonización versus infección, infección comunitaria versus infección intra-hospitalaria.

Aislado a incluir: El documento M39-A3 recomienda incluir sólo el primer aislado de una especie o género por paciente en el período analizado, sin considerar el sitio anatómico de obtención de la muestra, el patrón de susceptibilidad u otra característica fenotípica ${ }^{3}$. Esta metodología permite dar información relevante para definir terapias empíricas iniciales. Otros documentos plantean metodologías diferentes entre las que destacan: la inclusión de todos los aislados de un paciente, metodología que puede sobreestimar la resistencia dado que los pacientes con larga estadía tendrán múltiples cultivos. Si se utiliza el criterio basado en aislados por episodio se debe tener la precaución de homogenizar la definición de episodio y el intervalo de éste. Por último, se puede utilizar el criterio del primer aislado por fenotipo, en este caso se debe tener claro cuáles serán las características consideradas para diferenciar un fenotipo de otro ${ }^{5}$.

Información del estudio de susceptibilidad antimicrobiana: Es indispensable en este ámbito incluir las interpretaciones finales de las mediciones cuantitativas de concentración inhibitoria mínima (CIM) o diámetro de inhibición en el caso de difusión en disco, según las normas del CSLI correspondientes al año en vigencia; esto quiere decir utilizar las denominaciones de sensible (S), intermedio (I) o resistente (R). Registre los métodos de estudio de susceptibilidad empleado y las pruebas especializadas como por ejemplo confirmación de $\beta$-lactamasa de espectro extendido. La Tabla 1 muestra un ejemplo de recolección de datos.

\section{Procesamiento de los datos}

Después de recolectar los datos de acuerdo a los criterios establecidos, se debe proceder a su procesamiento considerando los siguientes elementos:

Verificar datos: Sólo incluya datos confirmados y chequee resultados inusuales como: Escherichia coli resistente a imipenem, Streptococcus pneumoniae resistente a ceftriaxona, Klebsiella pneumoniae resistente a amikacina y sensible a gentamicina, Enterobacter $\mathrm{sp}$ sensible a cefalotina, o investigue cualquier porcentaje de susceptibilidad que no sea $100 \%$ para antimicrobianos listados en el M100 sólo con criterio interpretativo de sensible. Por ej: Staphylococcus aureus resistente a vancomicina, nos orienta a errores en informes o de transcripción de datos. El documento CLSI M100-S19, en su Apéndice E, sugiere todos los resultados en que se debería verificar la susceptibilidad antimicrobiana o confirmar la identificación del microorganismo ${ }^{4}$.

Cautele que los datos estén realizados con puntos de

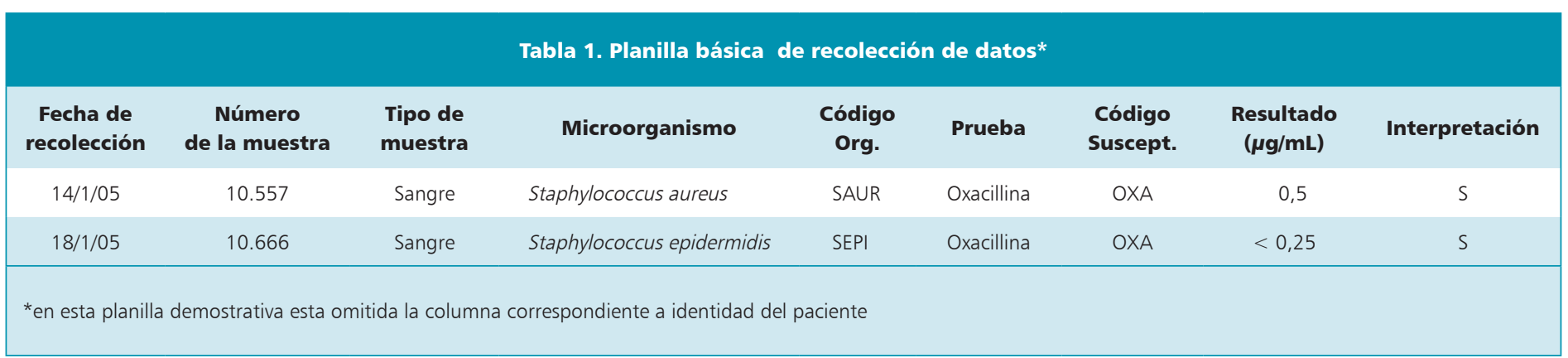


corte interpretativos de susceptibilidad antimicrobiana vigentes para el período estudiado. Si no es posible interpretar con puntos de cortes actualizados, entonces el reporte debe decir que los resultados no pueden ser comparables a lo largo del tiempo para aquellos antimicrobianos que han experimentado cambios en los puntos de corte. Se debe incluir una nota indicando el año (s) en que se modificaron los puntos de corte.

Considere sólo aquellos microorganismos con más de 30 aislados. Si estima esencial incluir una especie con menos de este número de cepas en un año, considere para ella un mayor intervalo de tiempo (por ej: 2 años) y coloque una nota explicando esta excepción.

Se recomienda incluir las siguientes especies, cuando el número de aislados es suficiente:

\section{Bacilos gramnegativos}

- Acinetobacter baumannii

- Citrobacter freundii

- Enterobacter sp

- Escherichia coli

- Haemophilus influenzae*

- Klebsiella pneumoniae

- Morganella morganii

- Proteus mirabilis

- Providencia sp

- Pseudomonas aeruginosa

- Salmonella sp

- Serratia marcescens

- Shigella sp

- Stenotrophomonas maltophilia

* Especies productoras de $\beta$-lactamasas reporte resultado como nota al pie de la tabla.

\section{Cocos grampositivos}

- Enterococcus sp*

- Staphylococcus aureus

- Staphylococcus coagulasa negativa

- Streptococcus pneumoniae

- Streptococcus grupo viridans (sólo aislados de sitios estériles)

* De preferencia consigne especies por separado si la identificación lo permite.

Antimicrobianos a incluir: Incluya sólo los antimicrobianos testeados rutinariamente. Reporte el antimicrobiano usado en el paciente y no los análogos para estudio de laboratorio. Por ej: cuando se utiliza el disco de cefoxitina para la detección de resistencia a oxacilina, reporte el porcentaje de susceptibilidad a cloxacilina. También se debe tener en cuenta que en algunos microorganismos los resultados de susceptibilidad se obtienen de hallazgos distintos a la medición de los halos de inhibición o de la CIM. Por ej: $S$. aureus en que se reporta resistencia a los $\beta$-lactámicos cuando son resistentes a oxacilina in vitro.

Con respecto a los antimicrobianos complementarios se debe tener cautela al incluirlos, dado que éstos sesgarán los resultados subestimando la susceptibilidad porque provienen de un subgrupo de aislados menos susceptibles, por ej: colistín frente a Pseudomonas aeruginosa multi o panresistente.

Cálculos: Se deben expresar los datos en porcentaje de susceptibilidad bacteriana, no incluyendo el rango I, con excepción de Streptococcus spp del grupo viridans y penicilina en que se sugiere informar ambos. También puede ser útil informarlo en $S$. pneumoniae dado que rangos intermedios pueden ser tratados con dosis altas de penicilina en infecciones no meníngeas. El porcentaje de I puede ir como nota.

Es ideal mantener los datos históricos de CIM o halos con la interpretación del período estudiado para ser comparados a lo largo del tiempo, sobre todo en el caso de haberse modificado los punto de cortes de interpretación.

Decisiones en la interpretación de la CIM: A continuación se mencionan recomendaciones en la interpretación correcta de CIM para el análisis de datos.

- Sobre valores decimales de CIM considere un potencial error interpretativo de los valores de CIM cuando los puntos de corte para S y/o R son menores de 1 . Por ej: valores de CIM menores o iguales a 0,06 son considerados susceptibles, mientras que valores de CIM 0,12 son considerados intermedios. Entonces, los laboratorios que guardan sus registros como 0,062 ó 0,064 pueden interpretar incorrectamente estos resultados como intermedios.

- En el caso de determinación de CIM utilizando diluciones de antimicrobianos, no mencionadas por la CLSI, deben ser consideradas y se les debe dar la interpretación del siguiente valor de dilución para CIM entregado por CLSI. Por ej: si el punto de corte para resistencia es 8 , entonces un valor de CIM de 6 debe ser registrado como tal pero tener la interpretación de resistente.

- Por último, en caso de valores de CIM fuera de rango, registre valores de CIM fuera de las diluciones utilizadas por ej. $>256$ ó $<1$. Si bien, los valores bajo el rango habitualmente caen en la categoría $\mathrm{S}$ y los valores sobre el rango en $\mathrm{R}$, la capacidad para interpretar estos valores fuera de rango se puede ver afectada si los puntos de corte cambian Por ej: un valor de CIM $>4$ se considera resistente si el punto de corte es 4 , si el punto de corte cambia a 16 , este valor de 4 no será interpretable según las nuevas recomendaciones. Entonces, el laboratorio puede reportar estos valores como "no interpretable con los nuevos criterios CLSI". 


\begin{tabular}{|c|c|c|c|c|c|c|c|c|c|c|}
\hline Microorganismo & n cepas & 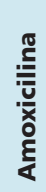 & 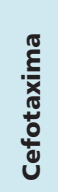 & 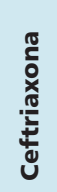 & 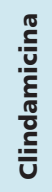 & 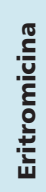 & 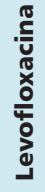 & 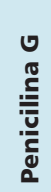 & 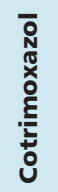 & 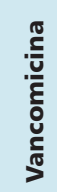 \\
\hline S. pneumoniae & 110 & 94 & - & - & 81 & 64 & 99 & - & 69 & 100 \\
\hline Meningitis & 110 & & $90^{1}$ & $90^{1}$ & & & & $85^{3}$ & & \\
\hline No meningitis & 110 & & $95^{2}$ & $95^{2}$ & & & & $92^{3}$ & & \\
\hline
\end{tabular}

${ }^{1}$ El punto de corte para sensible en pacientes con meningitis es $\leq 0,5 \mu \mathrm{g} / \mathrm{ml}$. ${ }^{2} \mathrm{El}$ punto de corte para sensible en pacientes con no meningitis es $\leq 1 \mu \mathrm{g} / \mathrm{ml} .{ }^{3} \mathrm{El}$ punto de corte para sensible en penicilina es $\leq 0,06 \mu \mathrm{g} / \mathrm{ml}$ para pacientes con meningitis y es $\leq 2$ en pacientes sin meningitis. En los casos de no meningitis los no susceptibles incluyen $7 \%$ de intermedios (CIM $4 \mu \mathrm{g} / \mathrm{ml}$ ) y $1 \%$ de resistentes (CIM $\geq 8 \mu \mathrm{g} / \mathrm{ml})$. En los casos de meningitis no existe la categoría intemedia

Validación de los resultados: Es importante validar los números totales y frecuencias para que se correspondan con la realidad y sean reproducibles; esto también es importante de realizar en caso de que las frecuencias o porcentajes sean demasiado disímiles de un año para otro. Para ello, seleccione las especies que tengan el menor número de aislados, imprima un listado o planilla con todos los aislados. Compare los resultados de estudios de susceptibilidad mostrados en la lista con el resultado realizado para el reporte. Verifique de esta forma el número total de cepas y el porcentaje de susceptibles para cada agente antimicrobiano. Esta es una forma fácil de detectar errores de traspaso de datos a planillas de cálculos, sobre todo si los registros son manuales.

Microorganismo/antimicrobianos específicos: A continuación se mencionan casos específicos de microorganismos que requieren un procesamiento diferente de los datos, de manera que permitan una mejor interpretación de los resultados en relación a la utilidad clínica de los antimicrobianos.

- Streptococcus pneumoniae: Frente a penicilina, cefotaxima y ceftriaxona calcule e incluya el porcentaje de $\mathrm{S}$ usando ambos puntos de corte, meníngeos y no meníngeos en todos los aislados, independientemente del sitio de origen (Tabla 2).

- Streptococcus spp grupo viridans: En caso de penicilina, registre porcentaje de S e I. Incluya datos de microorganismos aislados sólo de sitios normalmente estériles.

- Staphylococcus aureus: Puede ser útil analizar por separado la susceptibilidad de S. aureus sensibles (SAMS) y resistentes (SAMR) a oxacilina. Esto demostrará que SAMR es menos susceptibles a otros agentes anti-estafilocóccicos (Tabla 3).

- Enterococcus sp: Analice por separado la susceptibilidad de E. faecalis y E. faecium debido a los distintos patrones de susceptibilidad que presentan entre ellos. $\mathrm{Si}$ la identificación es sólo a nivel de género analice el grupo de género Enterococccus (Tabla 4).

Estratificación adicional de los datos: La estratificación adicional de los datos puede ser usada para responder preguntas que una institución de salud puede tener relacionada con la terapia antimicrobiana empírica en distintos escenarios o frente a situaciones clínicoepidemiológicas especificas. Para esto se debe determinar si hay un número suficiente de aislados para que los resultados que se obtengan en los subgrupos involucrados tengan una validez estadística.

\section{Presentación de los resultados}

Los resultados de los datos de susceptibilidad acumulada deben ser presentados en tablas y en un formato que permita la visualización amigable y práctica. Dentro de los aspectos generales es importante incluir el período de tiempo del estudio, el nombre del laboratorio, información de contacto de los responsables del informe y la metodología utilizada. Cuando el reporte es realizado por primera vez con esta metodología, se debe agregar una nota aclarando que se esta aplicando una nueva metodología para el análisis de los datos y que por lo tanto, la comparación con reportes anteriores debe ser cuidadosa. Existen aspectos específicos que se mencionan a continuación. 


\begin{tabular}{|c|c|c|c|c|c|c|c|c|c|}
\hline Microorganismo & n cepas & 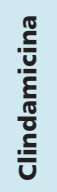 & 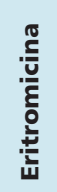 & 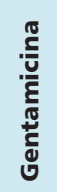 & 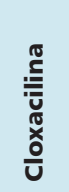 & 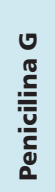 & 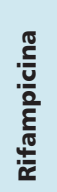 & 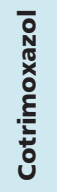 & 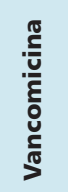 \\
\hline Todos los S. aureus & 1.317 & 80 & 50 & 93 & 68 & 13 & 98 & 96 & 100 \\
\hline S. aureus meticilina resistente & 449 & 44 & 4 & 84 & 0 & 0 & 97 & 95 & 100 \\
\hline S. aureus meticilina sensible & 904 & 97 & 74 & 96 & 100 & 18 & 99 & 97 & 100 \\
\hline \multicolumn{10}{|c|}{$\begin{array}{l}\text { Nota: en este ejemplo la suma de las cepas de } S \text {. aureus meticilina sensible y meticilina resistente es mayor que todos los } S \text {. aureus. Estc } \\
\text { se debe a que el primer aislado por paciente de los } S \text {. aureus meticilina sensible y resistente esta incluido en cada uno de los tres grupos } \\
\text { analizados, cuando un paciente tiene más de un tipo aislado. }\end{array}$} \\
\hline
\end{tabular}

\section{Aspectos relacionados con la construcción de tablas}

Confeccione tablas separadas para bacterias gramnegativas, grampositivas y anaerobios de importancia clínica, utilice un orden, ya sea alfabético, por grupo o según prevalencia. Presente por grupo o género si la información por especie no está disponible. Otra opción es agrupar varias especies dentro de un género (por ej: Shigella spp). Incluya el número de aislados para cada microorganismo, esto permite que el usuario pueda estimar con cierta precisión el valor neto del porcentaje de susceptibilidad. Con respecto a los antimicrobianos a utilizar en las tablas, use el nombre completo, si se prefiere abreviaciones deben estar explicitadas en glosarios al final del reporte o ser aceptadas por el documento M100 del CSLI. Ingrese datos a la tabla en porcentaje de susceptibilidad para cada

\section{Tabla 4. Porcentaje de susceptibilidad de Enterococcus spp}

\begin{tabular}{|c|c|c|c|c|c|c|}
\hline Microorganismo & n cepas & $\frac{\frac{\pi}{5}}{\frac{\bar{z}}{\frac{\sigma}{2}}}$ & 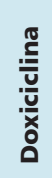 & 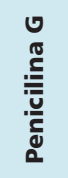 & 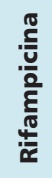 & 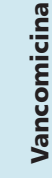 \\
\hline E. faecalis' & 7 & 100 & 84 & 100 & 64 & 95 \\
\hline E. faecium ${ }^{2}$ & 271 & 2 & 89 & 2 & 30 & 13 \\
\hline Todos los Enterococcus & 1.525 & 74 & 69 & 74 & 45 & 83 \\
\hline
\end{tabular}

${ }^{1} 16 \%$ de resistencia de alto nivel a gentamicina y estreptomicina. Interpretar con cautela, este grupo presenta número de aislados menor a $30 .{ }^{2} 55 \%$ de resistencia de alto nivel a gentamicina y estreptomicina microorganismo/antimicrobiano, ponga una raya en la celda de la tabla si el antimicrobiano no es testeado o es inefectivo clínicamente (por ej: combinación Proteus sp y nitrofurantoína).

\section{Otras opciones de presentación}

Variaciones en listados de antimicrobianos por sitio anatómico. Los laboratorios pueden seleccionar diferentes listados de antimicrobianos a ensayar para un mismo microorganismo en diferentes sitios anatómicos. Por ejemplo, un listado de agentes antimicrobianos puede ser testeado para aislados de E. coli de origen urinario y otro para no urinarios. En este caso, podría ser necesario reportar subgrupos, por ej: si levofloxacina fue testeada sólo en $E$. coli de origen no urinario, entonces se puede incluir en un pie de página que diga que los resultados de ese antimicrobiano son sólo interpretables para ese origen (Tabla 5) o simplemente consignar separadamente los resultados para ambos subgrupos (Tabla 6).

Localizaciones específicas. Los laboratorios podrían presentar el informe de susceptibilidad antimicrobiana en tablas separadas para las unidades de cuidados intensivos, u otra unidad específica. Los aislados de los pacientes de UCI son a menudo significativamente más resistentes.

Emergencia de resistencia. Se puede generar una tabla o gráfico con datos acumulados de varios años de manera de mostrar la evolución de la resistencia a un determinado antimicrobiano, o bien, evidenciar el momento de aparición de una nueva resistencia. A modo de ejemplo se pueden incluir:

S. aureus: porcentaje de S para oxacilina.

Enterococcus sp: porcentaje de $\mathrm{S}$ a vancomicina. 


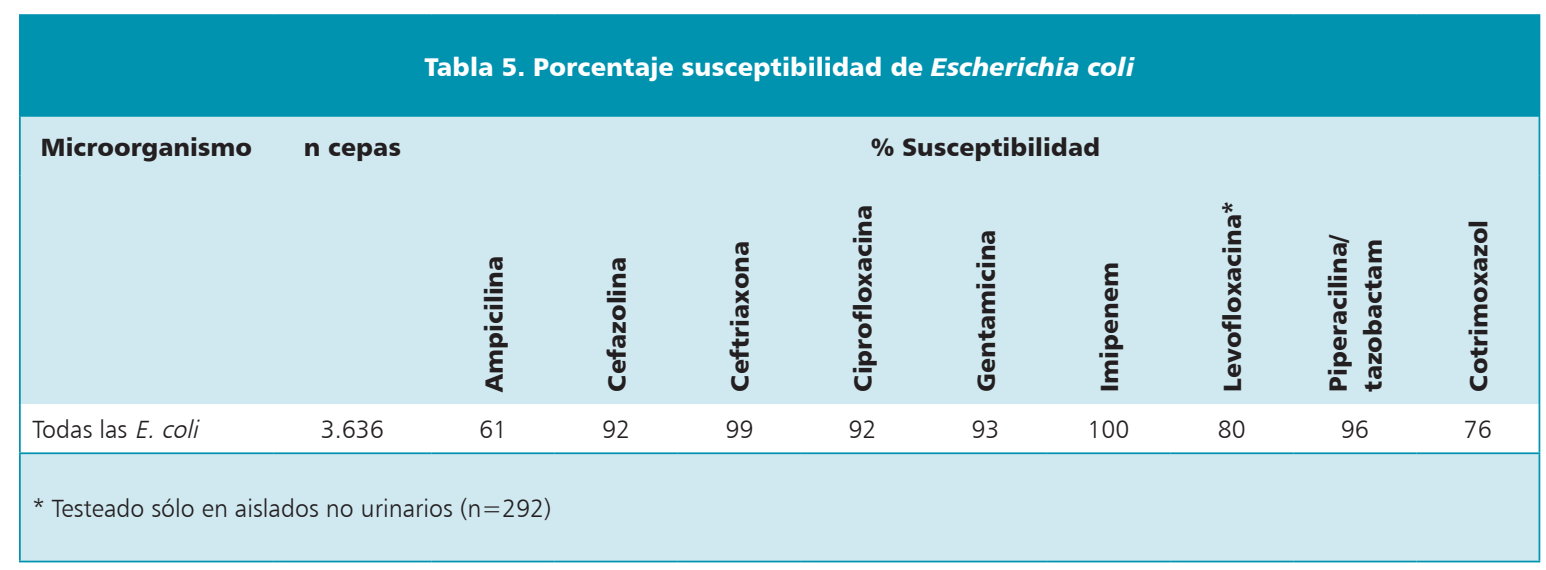

\begin{tabular}{|c|c|c|c|c|c|c|c|c|c|c|}
\hline \multirow{2}{*}{ Microorganismo } & \multirow[t]{2}{*}{ n cepas } & \multicolumn{9}{|c|}{$\%$ Susceptibilidad } \\
\hline & & 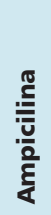 & 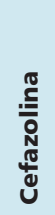 & 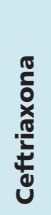 & 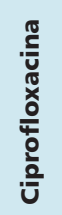 & 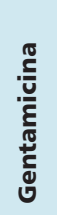 & 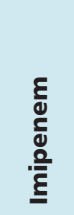 & 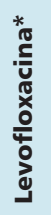 & 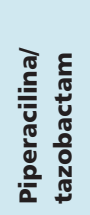 & 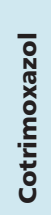 \\
\hline Todas las E. coli & 3.636 & 61 & 92 & 99 & 92 & 93 & 100 & 80 & 96 & 76 \\
\hline E. coli no urinarias & 292 & 44 & 82 & 96 & 80 & 87 & 100 & 80 & 93 & 62 \\
\hline E. coli urinarias & 3.417 & 63 & 93 & 99 & 93 & 94 & 100 & NT & 97 & 77 \\
\hline
\end{tabular}

K. pneumoniae y E. coli: porcentaje de aislados que producen $\beta$ lactamasa de espectro expandido-BLEE.

$P$. aeruginosa: porcentaje de $\mathrm{S}$ a fluoroquinolonas e imipenem.

Análisis de los resultados. Pueden realizarse múltiples comparaciones de los datos para buscar diferencias o tendencias en los rangos de susceptibilidad. Por ej.: comparación del año actual con años previos, pacientes intra-hospitalarios y extra-hospitalarios, comparación de la información de la institución con datos regionales o nacionales. Es útil calcular el intervalo de confianza 95 para ilustrar la precisión de los datos, dado que el tamaño muestral puede afectar la precisión de los datos.

\section{Uso del informe de susceptibilidad antimicrobiana}

El informe o reporte de susceptibilidad antimicrobiana puede ser utilizado como guía general para terapia antimicrobiana empírica inicial, hasta que los resultados específicos de susceptibilidad estén disponibles, el médico clínico puede usar, entonces, esta información como un elemento importante a considerar en la elección del antimicrobiano. Idealmente el informe debe ser de bolsillo, en un formato accesible para los médicos clínicos. Una tarjeta plegable laminada ha demostrado ser útil, como alternativa la institución puede decidir tener una versión extensa disponible en el sitio web.

\section{Difusión de la información}

La información recabada debe ser difundida localmente, destacando los resultados más importantes que ayuden a educar en relación con los patrones de susceptibilidad específica de la institución y las tendencias en el tiempo. Es importante explicar cuál es el propósito del informe y la metodología utilizada y si se ha monitoreado durante años es de utilidad presentar gráficos para mostrar tendencias. 


\section{Limitaciones de la información}

Limitaciones relacionadas con prácticas de indicación de cultivos: La calidad de la información se correlaciona con la realización de estudios microbiológicos. Si en la institución se solicitan pocos cultivos y frecuentemente los pacientes son tratados empíricamente, la información puede no representar verdaderamente los perfiles de susceptibilidad de los agentes etiológicos.

Limitaciones relacionadas con la influencia de un pequeño número de aislados: Debe informarse siempre el número de aislados por especie en el informe. Si el número de cepas es inferior a 30 en el período analizado, sea cauteloso en informarlo en el reporte, dado que su valor estadístico es limitado. En estos casos, se sugiere utilizar información de varios años, combinar información de especies de un mismo género, combinar información de varias instituciones de un área geográfica, o proveer información de publicaciones y guías.

Limitación en la identificación de nuevos patrones de resistencia: Cuando en los resultados se contempla sólo el primer aislado por paciente, se puede subestimar la información de emergencia de nuevos patrones de resistencia. Por ejemplo, un segundo o último aislado de $S$. aureus I a vancomicina, podría no aparecer en el resumen si el primer aislado de $S$. aureus era susceptible a vancomicina. Por lo tanto, la detección de nuevos o inusuales patrones de resistencia es preferible sea manejado como parte de la función diaria de verificación de resultados de antibiogramas, labor estratégica del laboratorio de microbiología.

A pesar de las limitaciones expuestas anteriormente, consideramos que el análisis y difusión de los resultados de susceptibilidad acumulada, debe ser considerado como una labor fundamental y sistemática del laboratorio de microbiología clínica en la actualidad, dado que aporta información de gran relevancia clínica y epidemiológica para las Instituciones de Salud.

\section{Resumen}

Considerando la gran variabilidad en la distribución de la resistencia microbiana, es una necesidad que cada centro de salud genere reportes locales de datos acumulados de susceptibilidad, con el propósito de guiar las decisiones clínicas y detectar tendencias que permitan establecer medidas de prevención para evitar la diseminación de cepas resistentes. Esta guía tiene como objetivo entregar recomendaciones para el análisis de susceptibilidad antimicrobiana y aportar datos útiles para la elaboración del informe local. Las recomendaciones que contenidas em este documento están basadas en el documento "Analysis and Presentation of Cumulative Antimicrobial Susceptibility Test Data de Clinical and Laboratory Standards Institute (CLSI) (3). Se describen aspectos claves relacionados con los requerimientos de la información, el procesamiento de los datos, el análisis y presentación de éstos.

\section{Referencias}

1.- Bax R, Bywater R, Cornaglia G, Goossens H, Hunter P, Isham V, et al. Surveillance of antimicrobial resistance: what, how and whither? Clin Microbiol Infect 2001; 7 : 316-25.

2.- Critchley I A, Karlowsky J A. Optimal use of antibiotic resistance surveillance systems. Clin Microbiol Infect 2004; 10: 502-11.

3.- Analysis and presentation of cumulative antimicrobial susceptibility test data; Approved guidelines-Third Edition. CLSI document m39-A3. Wayne,PA: Clinical and Laboratory Standards Institute; 2009.

4.- Performance Standards for Antimicrobial
Susceptibility Testing; nineteenth informational supplement. Volume 29, number 3; Clinical and Laboratory Standards Institute; 2009.

5.- Analysis and presentation of cumulative antibiograms: A new -consensus guidelines from Clinical and Laboratory Standard Institute. Hindler JF, Stelling J. Clin Infect Dis 2007: 44; 867-73. 